\title{
Grapes on the retina- a rare case of cavernous hemangioma of the retina
}

\author{
Bodhraj Dhawan*, Vipin Kumar Vig, Preetam Singh, Ankur Goel, Rajbir Singh \\ SB DR Sohan Singh Eye Hospital, Chowk Farid, Katra Sher Singh, Amritsar, Punjab, India-143006 \\ *Corresponding author E-mail: bodhrajdhawan@yahoo.co.in
}

Copyright $\odot 2014$ Bodhraj Dhawan. This is an open access article distributed under the Creative Commons Attribution License, which permits unrestricted use, distribution, and reproduction in any medium, provided the original work is properly cited.

\begin{abstract}
Cavernous Henangioma of the Retina is a rare, benign, congenital malformation which is non-progressive, usually unilateral, somewhat more frequent in women, and rarely a source of intraocular haemorrhage. Though the diagnosis is purely clinical, differentiation from other vascular anomalies particularly coats disease is important because of therapeutic implications. Systemic association's specifically cerebral involvement need to be ruled out on neuroimaging. Whereas Unnecessary laser treatment might at times precipitate a serious vitreous hemorrhage, a vitreous hemorrhege in a pregnant young female who otherwise had a good visual acuity, could at times point towards diagnosis of an undetected cavernous hemangioma of the retina.
\end{abstract}

Keywords: Cavernous Hemangioma of The Retina, Fundus Fluorescein Angiography.

\section{Case Report}

A 38 years old Indian female presented to us with complains of noticing Diminution of vision in her Right eye of one year back. She is a young non diabetic and non-hypertensive lady. Visual acuity was 20/120 not improving with pinhole in Right eye and 20/20 in the left eye.

Ocular examination findings were non remarkable in the left eye.

Anterior segment findings in right eye were unremarkable

Fundus of the Right eye showed Sessile grape like cluster of aneurysmal vascular dilatations on the posterior pole typically resembling "Bunch of Grapes".[Photo 1\&2].

Fundus Fluoroscein Angiography (FA) was done which revealed anurysmal vascular dilatations and delayed filling in the venous phase. FA typically revealed hypofluoroscent fluid levels due to separation of red blood cells and plasma as hyperfluoroscent.

Findings were consistent with the diagnosis of Cavernous Hemangioma of the Retina.[Photo 3,4,\&5] .MRI scan of the brain was essentially normal.

Patient was advised observation and annual ophthalmological examination to look for any progression. 


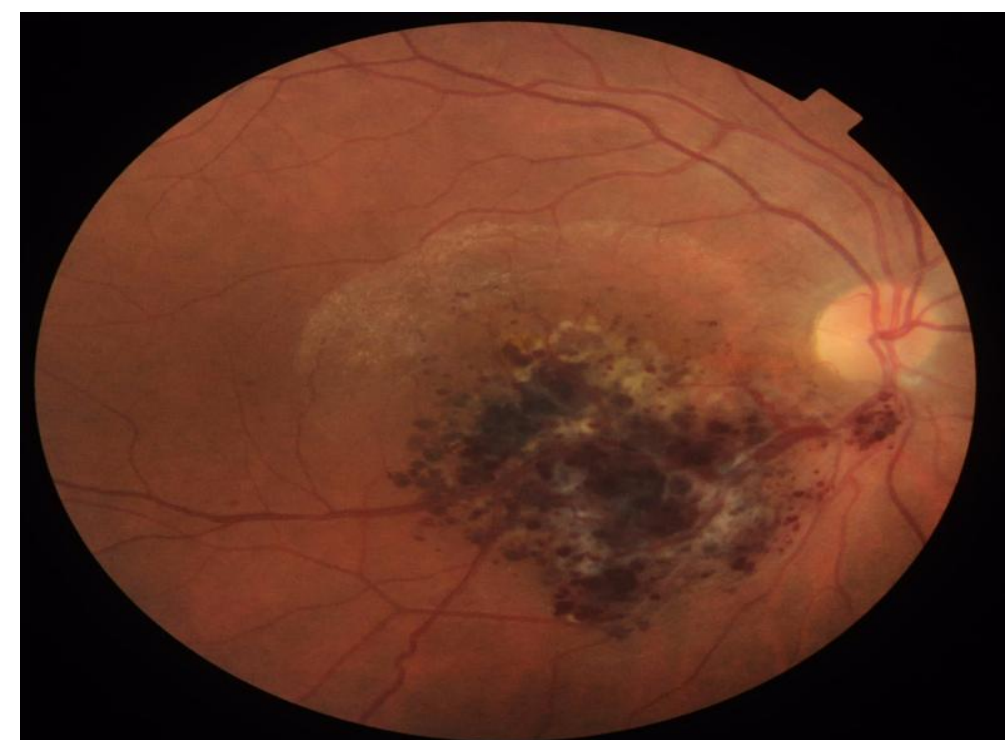

Photo. 1: Color fundus photograph of the Right eye showing multiple small aneurismal dilatations in clusters along the course of inferior temporal vein on the posterior pole. Note there is absence of any exudates, feeder vessels or surrounding subretinal fluid. The Lesion extends superiorly to invade macula.

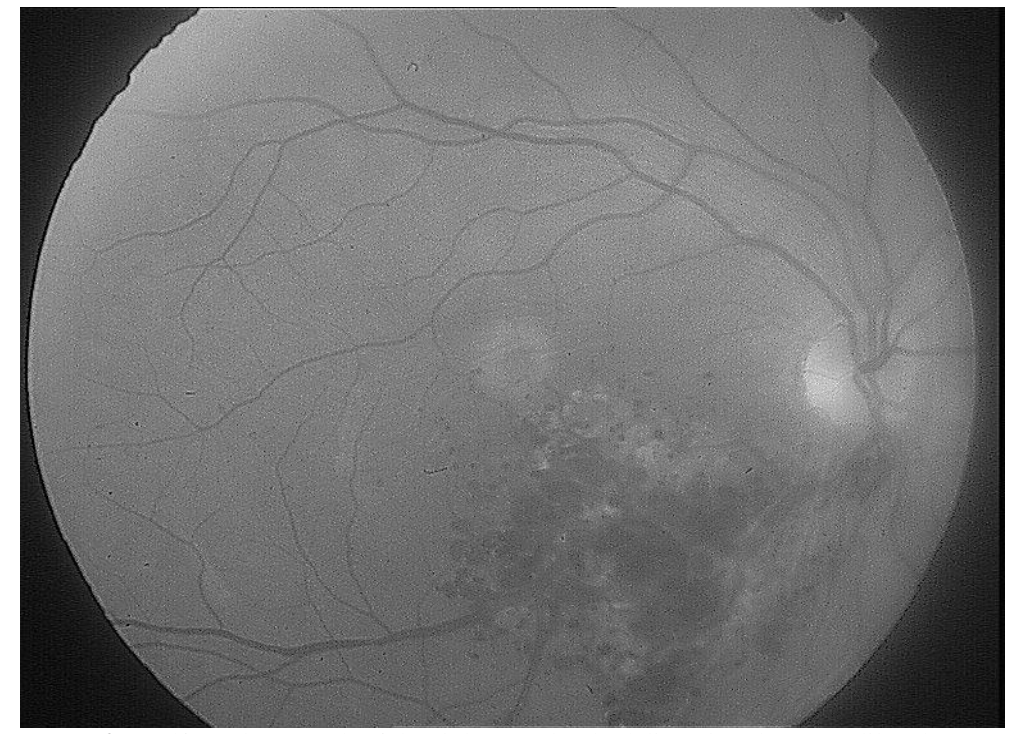

Photo. 2: Redfree photograph of the right eye clearly delineating the grape like clusters.

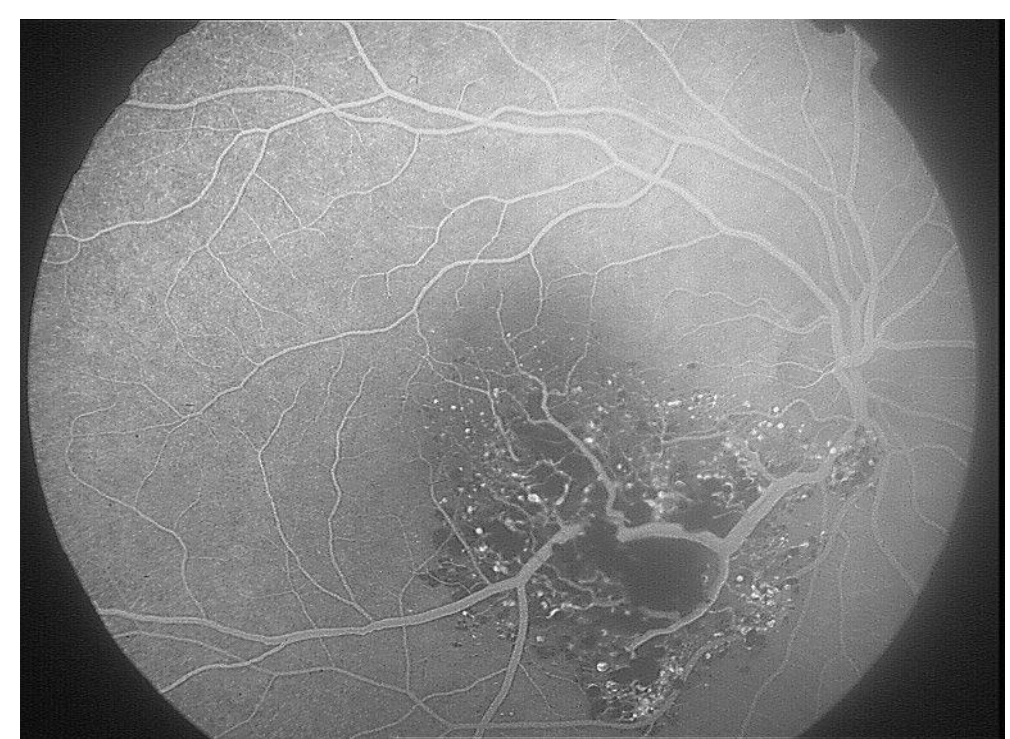

Photo. 3: Early phase Fluoroscein Angiogram of the Right eye shows that surrounding retina is nicely perfused but the venous drainage of the peripheral aneurysms is slowed. 


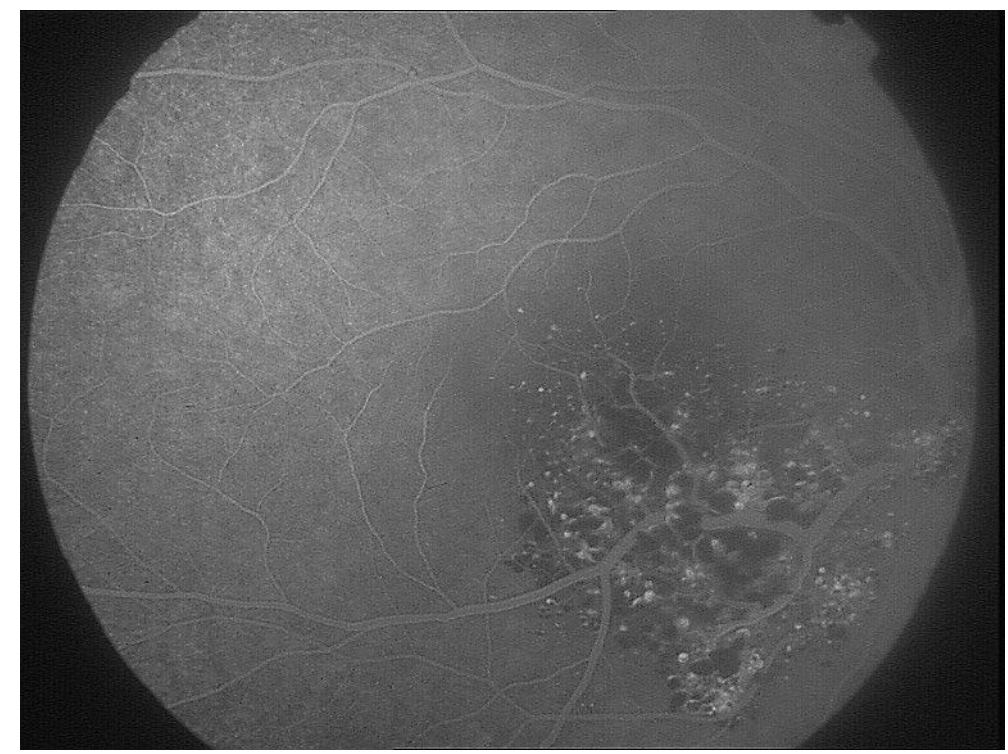

Photo. 4: After 3 minutes the surrounding retinal fluorescence begins to fade but more and more aneurysms fill and gain more fluorescence and more and more veins seen connecting with the main draining inferotemporal vein.

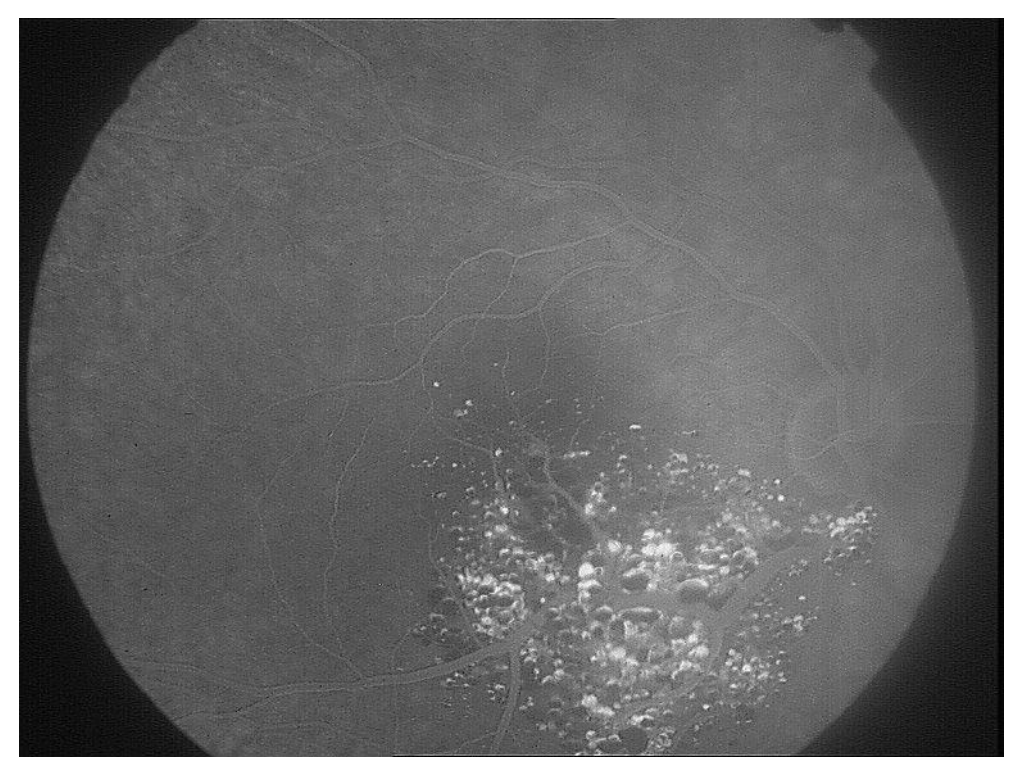

Photo. 5: Late phase of the angiogram shows plasma erythrocyte sedimentation within saccules as hypofluorocence of irregularity within the previously filled areas.

\section{Discussion}

Cavernous hemangiomas of the retina are rare congenital vascular hamartomas. [1]

In the first edition of his textbook, Reese (I95I) described a group of patients with 'capillary haemangioma of the retina'.[2] Later Gass (1 97I) described cavernous haemangioma of the retina as typically a localized, sessile, vascular tumour consisting of numerous, closely clustered, thin-walled globules sprouting from a retinal vein.[1]

Clinically, Patients may be asymptomatic, or they may present with decreased visual acuity depending upon the location of the tumor, presence of macular fibrosis, or vitreous hemorrhage. In a small series of nine patients, the mean age of presentation ranged from 1 to 55 years.[3]Whereas in a vast majority of cases the cavernous hemangioma may be an incidental finding on routine examinations , in a few it is detected when a vitreous hemorrhage is precipitated on valsalva maneuvers particularly in females during labor [4].

Ophthalmoscopically, retinal cavernous hemangiomas appear as a grouping of blood-filled saccules within the inner retinal layers or on the surface of the optic disc. [1]These tumors are often described as having a "cluster of grapes" appearance. The lesions are variable in size and location, and frequently follow the course of a major retinal vein. Epiretinal membranes are a common feature. In contrast to retinal capillary hemangiomas, cavernous hemangiomas lack prominent feeder vessels, intraretinal exudate, and surrounding subretinal fluid. Because the fundoscopic features are characteristic, diagnosis can generally be made based upon clinical appearance.[2].

The fluorescein angiographic features include a normal arterial and venous supply, extraordinarily slowed venous drainage, no arterio-venous shunting, no disturbances of vascular permeability, and no secondary retinal exudation. 
Almost always, isolated clusters of vascular globules with plasma/erythrocyte sedimentation surround the main body of the malformation. These findings differentiate the anomaly from other retinal vascular diseases.

Therapeutic intervention is seldom necessary.[5]However in cases where macula is involved with threat to vision, there is case report with infliximab given intravenously showing visual acuity improving with a marked involution of the tumor in the right eye. [6]

Although association of cavernous hemangioma of the retina has been shown with cerebral and other systemic hemangiomas, Patient's life is hardly damaged by complications of cavernous hemangioma of the brain. Because of autosomal dominant transmission all family members, with and without fundus findings, should undergo computed tomography or magnetic resonance imagining of the brain to preclude intracranial vascular anomaly. [7].

\section{References}

[1] Gass JD. Cavernous hemangioma of the retina. A neuro-oculo-cutaneous syndrome. Am J Ophthalmol 1971; 71:799-814.

[2] Reese A. B. (1951) 'Tumors of the Eye', Ist ed., p. 375. Hoeber, New York.

[3] Messmer E, Laqua H, Wessing A, Spitznas M, Weidle E, Ruprecht K, et al. Nine cases of cavernous hemangioma of the retina. Am J Ophthalmol 1983; 95:383-90.

[4] Smith BT, Joseph DP.Labor-induced hemorrhage of a retinal cavernous hemangioma. Ophthalmic Surg Lasers Imaging. 2009; 40(4):419-20.

[5] Richard Alan Lewis, M. Harry Cohen and George N. Wise Cavernous haemangioma of the retina and optic disc A report of three cases and a review of the literature Br J Ophthalmol 1975; 59: 422-434.

[6] Japiassú RM, Moura Brasil OF, de Souza EC. Regression of Macular Cavernous Hemangioma with Systemic Infliximab. Ophthalmic Surg Lasers Imaging. 2010; 9:1-3.

[7] Skoumalová S, Boguszaková J Retinal cavernous hemangioma associated with intracerebral cavernous hemangioma in a 33-year follow-up study Cesk Slov Oftalmol. 1999; 55(5):316-22. 